\title{
LEAP-GWU-2015: Centrifuge and numerical Modelling of slope liquefaction at the University of Cambridge
}

\author{
Srikanth S. C. Madabhushi ${ }^{1}$, Stuart K. Haigh ${ }^{1}$, Gopal S. P. Madabhushi ${ }^{1}$
}

\begin{abstract}
:
As part of the LEAP-GWU-2015 exercise, a dynamic centrifuge test was conducted at the University of Cambridge on a $5^{\circ}$ slope of medium dense Ottawa F-65 sand. The model preparation and saturation details are presented in this paper. This paper presents the experimental data recorded during small and large magnitude sinusoidal ground motions. After the experiment, numerical simulations of the experiment were performed using the finite element code Swandyne. The results from these numerical analyses are compared with the centrifuge test data and the deformations observed during the post-test investigations. The numerical analyses replicated many of the salient features of the test, such as the overall generation of excess pore pressures and attenuation of accelerations in the liquefying ground. More subtle results, such as the de-liquefaction shocks and the asymmetric response due to diff erences in upslope and downslope accelerations were less well captured in terms of the expected spikes in the dynamic excess pore pressures and accelerations. Overall, the combination of centrifuge testing and numerical analysis were found to complement each other well
\end{abstract}

\section{Introduction}

Earthquake induced soil liquefaction continues to be an active topic of research, primarily driven by the continued observations of liquefaction induced failures in recent earthquakes such as the Tōhoku earthquake in Japan in 2011 and the Christchurch earthquakes of 2010 and 2011. The widespread liquefaction caused by the latter event and the damage it caused to important infrastructure such as bridges was considered by many researchers, for example, Haskell et al (2013).

Dynamic centrifuge modelling has been established as a useful way to recreate liquefaction under laboratory conditions since the early 1980s. The original VELACS project in the early 1990s funded by the National Science Foundation, USA was the first effort to use centrifuge modelling test data to compare with and validate the numerical procedures available at that time (Arulanandan and Scott, 1993). Since that time, the experimental techniques used in centrifuge modelling and the instrumentation employed have improved significantly. Similarly, the numerical procedures that are currently available to carry out fully coupled analysis and the computational power to perform such analyses have vastly improved.

There is a renewed interest amongst researchers and geotechnical engineering practitioners in many countries in developing a new database of centrifuge test data on liquefaction problems. Accordingly, a project named LEAP (Liquefaction Earthquake Analyses Projects) was organised, involving researchers from the US, Japan, China, Taiwan and the UK. As an exemplar problem, the case of fully saturated, sloping ground with a slope angle of $5^{\circ}$ was modelled at universities in Cambridge, Kyoto, Davis, RPI, Taiwan and China all of whom used the same Ottawa F-65 sand in their centrifuge tests. Numerical analyses of the same problem were also attempted elsewhere.

In this paper, the results from the centrifuge test carried out at Cambridge will be presented along with some of the unique features of the test set-up used. The Cambridge test was carried 
out at $40 \mathrm{~g}$. In addition to the experimental results, numerical analyses were also carried out using the fully coupled FE code Swandyne, (Chan, 1988). The results from the numerical analyses will be compared with the experimental results in this paper.

The full specifications of the slope test are reported by Kutter et al (2016) in the companion paper. The five required ground motions, of which three were small non-destructive ground motions and two were large magnitude ground motions, are also detailed. The reasoning behind the use of a rigid model container and the input motions specified are explained in that paper and will therefore not be repeated here.

\section{Model preparation}

The specification of these tests called for the use of a rigid model container in order to achieve numerically simple boundary conditions. At Cambridge, a transparent sided box with a Perspex window was used as the model container.

Air pluviation was used for the preparation of the model slope. An automatic sand pourer (Madabhushi et al, 2006), which is a spot pluviator, was employed to create a level soil bed as shown in Fig. 1. The achieved density of $1581 \mathrm{~kg} / \mathrm{m}^{3}$ was approximately $5 \%$ lower than the prescribed density of $1652 \mathrm{~kg} / \mathrm{m}^{3}$. The instruments were placed at the required locations during the construction of the soil model. Additionally, dry spaghetti strands were installed vertically near the front of the model slope. The spaghetti strands soften on saturation and were expected to move with the slope following liquefaction. Once the model was completed, it was saturated under vacuum using $40 \mathrm{cSt}$ methyl cellulose fluid to satisfy the dynamic scaling laws (Schofield, 1981) such that the rate of excess pore pressure generation due to earthquake loading scales in the same way as the rate of dissipation in the post seismic period. More details of this are given by Kutter et al (2016).

Prior to saturation, the soil model was flushed with $\mathrm{CO}_{2}$ gas in several cycles. This removes the air from the soil model and ensures a high degree of saturation. The saturation process was carried out using the CAM-SAT system that controls the rate of fluid mass flux into the base of the model, (Stringer and Madabhushi, 2009). The controlled mass flux of $\sim 0.5 \mathrm{~kg} / \mathrm{hr}$ prevents any fluidisation of the soil bed during saturation whilst maintaining the required driving potential head. The layout of the CAM-SAT system is shown in Fig. 2.

A simplified model cross-section of the Cambridge centrifuge model is shown in Fig. 3 with only the instruments that will be considered in this paper. In this figure it can be seen that the model slope surface has a logarithmic spiral shape. As the shaking direction is tangential to the motion of the centrifuge package, this shape has been adopted to correct for the curvature of the centrifugal acceleration field. An equivalent prototype slope therefore will have a planar sloping surface under 1-g conditions where the gravity field is effectively vertical. The logarithmic spiral describing the shape of the slope can be derived starting with the polar coordinate notation presented in Fig. 3. The variation in the radius over a small angle $d \theta$ can be written as:

$$
d r=r \beta d \theta
$$

Reorganising and integrating: 


$$
\begin{aligned}
& \int \frac{1}{r} d r=\int \beta d \theta \\
& \mathrm{r}=c e^{\beta \theta}
\end{aligned}
$$

The constant $c$ is determined based on the geometry of the centrifuge and the model. In order to achieve the logarithmic slope profile two guide plates were machined and attached to the top of the model container as seen in Fig. 4. This profile was used to shape the slope surface using a vertical cutting plate whose edges were moved along the guide plates. A flat soil model was first fully saturated using the process detailed above and then the fluid level was lowered to below the lowest depth of the cut required. The resulting capillary suction in the soil allowed the curved slope to be cut more easily and to hold its shape. This was considered preferable to cutting or vacuuming the dry soil bed as the subsequent saturation could have disturbed the curved slope geometry. The slope was then fully re-submerged under the fluid following the loading of the model onto the centrifuge.

\section{Cambridge Servo-Hydraulic Earthquake Actuator}

Recently, a new servo-hydraulic actuator that exploits the unique features of the $10 \mathrm{~m}$ diameter Turner Beam centrifuge at Cambridge has been developed. This new servo-hydraulic actuator has been used to apply the accelerations to the model slope tested in this project. Servo-hydraulic actuators theoretically have numerous benefits over previously used mechanical actuators, such as allowing for complex earthquake motions to be more accurately recreated, (Madabhushi et al., 2012). Kutter et al (2016) presents a comparison between the specified and achieved base motions in terms of the recorded time history and spectral acceleration. It was observed that the accelerations generated by the Servo-hydraulic actuator in these tests featured unspecified high frequency components though the resulting displacement very closely matched the specified input motion. The possible significance of these higher frequency components is investigated in section 9.

The target motions were prescribed for all the centrifuge centres a priori. These were sinusoidal ramp motions as described by Kutter et al. (2016). The peak amplitudes were prescribed for Motions \#1 and \#2 as $0.015 \mathrm{~g}$ and $0.15 \mathrm{~g}$ respectively.

\section{Transit of the actuator}

During operation of the servo-hydraulic shaker, the actuator is initially positioned in a central position to allow for maximum displacement on either side, depending on the demanded earthquake. Therefore, before and after each earthquake event the shaker transits approximately $10 \mathrm{~mm}$ from the park position to the centre and vice versa. An example of the translational motion is shown in Fig. 5 for Motion \#2 that was fired on the model slope during this centrifuge test. In this figure the top trace shows the transit to centre position from -4 sec to -2 sec and its return to park position after the earthquake event from $5 \mathrm{sec}$ to $7 \mathrm{sec}$. The bottom trace in Fig. 5 shows the actual displacement achieved by the servo-hydraulic actuator during the ground motion that was measured directly from the LVDT mounted in the actuator. It must be pointed out that these values are all presented at model scale.

\subsection{Accelerations during transit}


In Figs. $6 \mathrm{a}$ and $6 \mathrm{~b}$ the accelerations recorded on the model container and within the soil slope during the transit phases of Motion \#1 and \#2 are presented. The data presented in this paper have been filtered using an $8^{\text {th }}$ order Butterworth low pass filter with a cut-off frequency of $150 \mathrm{~Hz}$. The original sampling frequency was $4 \mathrm{kHz}$ per channel. Again these are presented at model scale as recorded directly by the instruments. The accelerations during transit are relatively small compared to the accelerations during Motion \#2. However, some amount of amplification of these motions occurs as the stress waves propagate through the soil slope as recorded by AH1 and AH3 (see Fig. 6b). In the LEAP project, very small nondestructive earthquakes were additionally specified to allow for characterization of the soil following the destructive earthquakes (Kutter et al, 2016). In such a case, the transit accelerations may be larger than the ground motion, as shown in Fig. 6a. However, the transit accelerations did not result in any excess pore pressures, as discussed in Section 4.2. This suggests the transit accelerations were also not destructive in this case.

\subsection{Excess pore pressures generated during transit}

It is both important and instructive to see what excess pore pressures are generated during the transit phase. For this purpose the excess pore pressures recorded during Motions \#1 and $\# 2$ are presented in Fig. $7 \mathrm{a}$ and $7 \mathrm{~b}$ respectively. In both cases it can be seen that no excess pore pressures are generated during the transit of the actuator from the park position to the central position, i.e. the accelerations in the soil body are too small to generate any significant volumetric strains. At locations P1 and P3 large excess pore pressures are generated during motion 2 between 0 and $1 \mathrm{sec}$, which then start to dissipate after the main ground motion event. However, no discernible change in the excess pore pressures was recorded during motion 1. It is very interesting to note the difference between Fig. $7 \mathrm{a}$ and $7 \mathrm{~b}$ as the actuator returns from its central position to the park position, i.e. between $5.5 \mathrm{sec}$ and $7 \mathrm{sec}$. Whilst in Fig. $7 \mathrm{a}$ there is again no change in excess pore pressures, Fig. $7 \mathrm{~b}$ shows that the transit accelerations can cause a rebuild up of excess pore pressures. It should be noted that the magnitude of the transit accelerations are similar between tests and between the travel to the centre and back. The reason for this behaviour is thus likely due to the fact that the excess pore pressures from the Motion \#2 had not fully dissipated by $5.5 \mathrm{sec}$ leading to lower effective stresses in the slope. These lower effective stresses can result in larger cyclic shear strains that triggers the generation of additional excess pore pressures.

\section{Finite element analysis}

In addition to the centrifuge tests, finite element analyses were carried out using the fully coupled code Swandyne, (Chan, 1988) that solves the Biot's equations with the $u-p$ formulation (solid displacement-fluid pressure). The detailed implementation of this code is described elsewhere, (e.g. Madabhushi and Zeng, 1998), and will therefore not be repeated here. A static run is first carried out to establish the geo-static stresses within the soil slope. This also establishes the hydrostatic pore pressures in the fluid phase. The final effective stress state from the static run is used as the initial stress state for the dynamic run. It must be pointed out that these FE analyses were carried out after the results from the centrifuge test were known.

\subsection{FE mesh and Boundary conditions}

The FE mesh was created using a constant slope that will represent the centrifuge model slope described in Fig. 3 earlier. The constant slope was used as the centrifuge model represents a straight slope at the prototype scale where the gravity field is always applied in 
the vertical direction. Iso-parametric elements with 8 solid nodes and 4 fluid nodes were used. The FE mesh consisted of a total of 375 elements with 832 solid nodes and 416 fluid nodes. The element sizes were determined based on the criteria laid out by Semblat et al (1999). They proposed that the element size in the direction of the shear wave propagation should be less than one tenth of the wavelength. The tallest element in the mesh in these analyses had a height of $0.36 \mathrm{~m}$. The highest frequency in the input signal was of the order of $3 \mathrm{~Hz}$ which implies accurate transmission of shear waves in liquefying soils with a shear wave velocity as low as $10.8 \mathrm{~ms}^{-1}$. The minimum time step required for the dynamic analyses was calculated following the recommendations outlined by Haigh et al (2005) for liquefaction analyses.

The base nodes of the FE mesh were all fixed and the input accelerations are applied to these base nodes. Following the centrifuge model, the horizontal input acceleration is also applied to the side boundaries with no fixity in the vertical direction. This simulates a rigid container whilst allowing the soil slope to settle during the application of the body forces during the static run, without 'hanging' from the side boundaries. A further fluid boundary condition is required at the soil surface. For the fluid nodes along the slope surface, the hydrostatic fluid pressure was calculated depending on their depth below the water table and the fluid node was held at this pressure. In addition to this, the solid nodes also require a vertical force based on the fluid pressure along the element they connect. This was calculated using an internal function within the Swandyne code. The correctness of the fluid pressure boundary was confirmed by checking the initial static distribution pore pressure and vertical effective stress contours throughout the soil slope. A horizontal distribution of hydrostatic pore pressures with a series of vertical effective stresses running parallel to the slope were correctly obtained.

\subsection{PZ Mark III model and constitutive parameters used}

In these analyses the PZ Mark III constitutive model was used as described by Pastor et al (1985). This is a bounding surface plasticity model that uses potential surfaces which are of the same family of curves as the yield surface. The non-associative behaviour of sands is captured by this model. The full description of the model and its implementation into Swandyne are described by Zienkiewicz et al (1999). This model has been shown to capture the cyclic behaviour of sands that are susceptible to liquefaction in many publications (e.g. Madabhushi and Zeng, 1998, Haigh et al, 2005, Coelho 2007 \& Madabhushi and Madabhushi, 2014).

As a starting point for the numerical analyses, a set of constitutive parameters were obtained primarily from Haigh et al (2005), with the exception of $\mathrm{M}_{\mathrm{f}}$ and $\mathrm{M}_{\mathrm{g}}$ which were obtained using the recommendations from Coelho (2007) for loose sands. In the referred work, the values were based on previous experience with other silica sands, such as Fraction E Leighton Buzzard sand or Hostun Sand, and calibrated against centrifuge data. For future analyses, the results from cyclic triaxial testing of Ottawa F-65 sand would help refine the parameters selected. The key constitutive parameters used in these analyses are summarised in Table 1.

\subsection{Rayleigh Damping}

An important consideration when modelling the response of saturated soils to dynamic events is how the energy imparted by the input motion is dissipated or damped by the numerical model. Soil constitutive models will often exhibit a degree of hysteresis which will damp input signals. In order to avoid specious oscillations in numerical solutions, particularly 
at small strains, additional non-physical damping is often introduced. Rayleigh Damping, as described by Clough and Penzien (1993) for example, is implemented in Swandyne and is defined by two parameters. The first parameter $\alpha$ is multiplied with the mass matrix of the system and $\beta$ is multiplied with the initial stiffness matrix. However, the relative contribution to the damping ratio of the mass proportional and stiffness proportional damping also depends on the frequency content of the oscillations. The contribution to the damping ratio from $\alpha$ decays with the reciprocal of the frequency whilst the contribution from $\beta$ increases linearly.

In numerical studies of liquefaction this is significant as the stiffness proportional damping decreases as the soil liquefies. The total damping ratio thus tends to that given solely by the mass proportional damping. Due to the decay of the mass proportional damping at higher frequencies, inappropriate selection of the damping parameters can potentially lead to nonphysical high frequency oscillations in the numerical solutions.

In this work, values of $\alpha=3$ and $\beta=0.005$ were chosen after running a series of analyses and selecting the best match of the accelerations with the experimental data. This is required as it is difficult to derive values of $\alpha$ and $\beta$ based on the physical soil model. The frequency dependence of the damping ratio implied by these parameters is shown in Figure 8. The same total damping ratio can be achieved by any number of combinations of $\alpha$ and $\beta$. However, the degradation of the soil stiffness meant that combinations with larger values of $\alpha$ were found to be preferable. In this figure it can be seen that close to the driving frequency of $1 \mathrm{~Hz}$ the damping ratio is about $20 \%$. Damping ratios of the order of $10-30 \%$ have been used previously by Dewoolkar et al. (1999) in liquefaction studies. It must also be noted that a degree of material damping is provided by soil hysteresis, though this will decrease as the soil liquefies.

\section{Slope response during small ground motion - Motion \#1}

The first target motion to be fired on the centrifuge model slope after reaching $40 \mathrm{~g}$ was Motion $\# 1$, which featured a peak demanded acceleration of $0.015 \mathrm{~g}$. The results from this ground motion are discussed first, alongside the results from the finite element analysis. The experimentally measured input motion was applied to the base nodes of the finite element mesh.

\subsection{Acceleration - time histories}

The acceleration time histories recorded by the central array of accelerometers are presented in Fig. 9 at prototype scale. Some of the instruments in this event have picked up some spurious accelerations as seen in the case of AH1 and AH4. For example, the acceleration shown by AH1 at a time of $10 \mathrm{~s}$ is before the shaking had commenced and is likely due to electrical noise during data logging. Overall the accelerations were very small and clear transmission of the shear waves without much attenuation was observed. The results from the FE analysis fit the experimental data at these locations very well. A slight difference is that the accelerations at $\mathrm{AH} 4$ are amplified in the numerical analysis.

\subsection{Excess pore pressure - time histories}

During Motion 1 not much excess pore pressure generation was expected as the input motion was small, resulting in only modest cyclic shear strains in the soil which fall below the threshold cyclic strain required to generate excess pore pressures. The base motion recorded by AH11 and the excess pore pressures recorded by P1 to P4 are presented in Fig. 10. This figure confirms that the pore pressure transducers P1 to P4 all record very little excess pore 
pressures. The finite element analysis also predicts very little excess pore pressures during this event as seen in Fig. 10. However, there is a slight accumulation of excess pore pressures during the analysis starting around $18 \mathrm{~s}$ when the peak acceleration was applied. The largest magnitude is at $\mathrm{P} 4$ and is of the order of $5 \mathrm{kPa}$ at the end of the analysis. This is consistent with the excess pore pressures draining vertically upwards. The predicted accumulation of small excess pore pressures with small ground motions which are not observed in the centrifuge may be due to the ability of the physical model to undergo small, localised volumetric strains in the soil skeleton and allow local redistribution of small excess pore pressures. This cannot be captured by the numerical analyses. Further, the constitutive parameters shown in Table 1 were calibrated against centrifuge data subject to large ground motions and thus may be less appropriate for predicting excess pore pressures in small ground motion events (Haigh et al, 2005).

\section{Slope response during large ground motion - Motion \#2}

The second ground motion that was applied to the slope model was reasonably large with a target peak acceleration of $0.15 \mathrm{~g}$. This ground motion is anticipated to generate large excess pore pressures thereby causing the $5^{\circ}$ slope to suffer lateral spreading. The results recorded in the centrifuge model during this event are presented below. Again, the finite element analysis was carried out by applying the recorded input motion to the base and side boundary nodes of the finite element mesh. The results from the numerical analysis are overlaid on the experimental results.

\subsection{Acceleration - time histories}

In Fig. 11 the acceleration-time histories for the central array of accelerometers AH1 to AH4 are presented. The locations of these instruments can be seen in Fig. 3. The experimental data clearly shows that the accelerations attenuate significantly, particularly in latter cycles as the stress waves propagate from the base towards the surface of the slope. This is anticipated as the soil stiffness will degrade with the onset of excess pore pressure generation thus reducing the transmission of stress waves through the soil body. Further, the accelerations in the shallow depths show 'spikes' that correspond to dilation of the soil causing the excess pore pressures to decrease. This is now a well-known phenomenon in liquefying soils, these spikes being referred to as 'de-liquefaction shocks' following Kutter and Wilson (1999). The numerical analysis also predicts attenuation of the acceleration comparable to that observed in the centrifuge results. In particular, the match of the accelerations at depth, such as that shown by AH1 is very good. At shallower depths the predicted accelerations appear to be over attenuated, and are less able to capture the sharp de-liquefaction shocks observed in the experimental results. The accelerations traces must be viewed in conjunction with the excess pore pressures at the corresponding locations, as discussed in Section 7.2.

\subsection{Excess pore pressure - time histories}

The excess pore pressure-time histories recorded during the motion 2 along the central array of pore pressure transducers are presented in Fig. 12. The predictions from the numerical analysis at the same locations are overlaid onto these plots. The centrifuge test data shows that the excess pore pressure rises rapidly at locations P1, P3 and P4. Unfortunately the pore pressure transducer at location $\mathrm{P} 2$ did not function in this centrifuge test. The magnitude of excess pore pressures decrease from about $40 \mathrm{kPa}$ at location $\mathrm{P} 1$ to about $8 \mathrm{kPa}$ at location P4. The numerical analysis is able to predict the final value of excess pore pressures quite well at all depths. The analyses also show some shear induced dilation in the soil which manifests as a drop in excess pore pressure during strong loading cycles. The corresponding 
accelerations at shallow depths hence show a degree of asymmetry, showing a difference between the upslope and downslope accelerations. The magnitudes of these drops are, however, smaller than those of the experimentally recorded drops in excess pore pressure. This is consistent with the observation in Section 7.1 that the predicted accelerations at AH1 and $\mathrm{AH} 2$ featured less discernible de-liquefaction shocks than the experimental results.

The amount of dilation depends on the direction of the loading and plastic flow vectors, which in Swandyne are controlled by four shape factors. These are the slopes of the critical state lines for the yield surface and plastic potential, and two parameters which relate dilatancy to the stress ratio for the loading vector and plastic strain vector, i.e. $\mathrm{M}_{\mathrm{f}}, \mathrm{M}_{\mathrm{g}}, \alpha_{\mathrm{f}}$ and $\alpha_{\mathrm{g}}$ as described in Table 1 (Zienkiewicz et al, 1999). The absolute amount of excess pore pressure generation also depends on the ratio of $\mathrm{M}_{\mathrm{f}}$ to $\mathrm{M}_{\mathrm{g}}$, and previous use of this constitutive model has suggested a trade-off between accurate prediction of the magnitude of excess pore pressures generated and capturing strong dilation and the associated deliquefaction shocks. Overall, the match between the predicted excess pore pressures and experimental observations is considered quite satisfactory, though the importance of centrifuge tests in highlighting the nuanced behaviour of sands is exemplified.

\subsection{Contours of excess pore pressure ratio}

In order to better understand the extent of liquefaction across the slope, contours of the excess pore pressure ratio can be constructed using the full set of PPTs installed in the model as shown in Fig. 3. Kutter et al (2016) describes the inclusion of transducers P9 and P10 for assessment of boundary affects. Fig. 13a and 13b show the contours calculated during and sometime after Motion \#2, in which the original instrument locations were assumed in calculating the initial confining stress. The contours shown are only interpolated between where instruments were placed. Having achieved reasonable predictions of the excess pore pressures using the numerical analyses as discussed in section 7.2, the experimental contours are complemented by the numerical predictions of the excess pore pressure ratio across the whole model.

At a time of $12 \mathrm{~s}$ the experimental contours indicate that the soil at shallow depths at the middle of the slope is approaching full liquefaction. The deeper soil exhibits a lower excess pore pressure ratio at this time. This may be physically rationalised as the deeper soil experiences a larger confining stress which would necessitate either larger shearing or more cycles to liquefy. The numerically derived contours also indicate that the deeper soil is less liquefied at this time.

Post-earthquake contours are plotted in Fig. 13b at the time instant of 26 s. Both the experimental and numerical contours indicate less liquefaction at the base than near the surface. This is due to the upwards drainage of pore fluid. The numerical model also shows that the slope has suffered lateral spreading. This causes the top left edge elements to go into tension and the top right edge elements into compression. This led to a substantially lower excess pore pressure ratio at these locations as seen in Fig. 13b. The solution at the middle of the slope where the main instrumentation arrays were located is presumably less affected by these errors. This presumption is consistent with the results of the numerical analysis. 


\section{Deformations in the slope}

One of the key aspects of the liquefaction of sloping ground is the accumulated deformations that can occur during and following earthquake loading. The amount of accumulated deformation in the centrifuge model was observed during the post-test excavation of the model as shown in Fig. 14a. In this figure the lateral movement can be detected by observing the shape of the spaghetti strands placed in the model at the time of its preparation. This can be compared with the deformed mesh at the end of Motion \#2 from the numerical analysis (see Fig. 14b). Although this is a slightly unfair comparison as the centrifuge model has seen further Ground Motions \#3, \#4 and \#5, of these only \#4 was considered destructive as described by Kutter et al (2016). Further, it was observed that after Motion \#2 the slope had spread and flattened substantially and comparisons with the post-test measurements showed little additional change in the slope geometry.

In Fig. 14b the original slope shape before the earthquake loading is shown. Interestingly, while the slope suffers settlement on the left half of the model the right half of the slope shows heaving, indicating a broad rotation of the slope material from the crest to the toe. The central point on the surface of the slope therefore suffers no settlement. This is consistent with the analysis being largely undrained during the first 30 seconds. This is confirmed by the excess pore pressure contours in Fig. 13, that show the dissipation of excess pore pressures is just beginning to take effect by this time. The amount of lateral spread seems to be greatest in the middle of the slope in Fig. 14b with only a small amount of lateral spread at the side boundaries. This aspect was also observed in the centrifuge slope in Fig. 14a where the spaghetti in the middle of the slope spread more than those in the ends. These observations were caused by the rigid boundaries in both cases.

\section{Influence of Input Motion}

Kutter et al (2016) presents a comparison between the base accelerations generated by the earthquake actuators at the different facilities. For the Cambridge Servo hydraulic shaker, the achieved displacements and velocities were found to match the prescribed traces reasonably well. However, the accelerations imparted to the model had additional unspecified high frequency components. Later in the paper, it is also observed that the extent of liquefaction was greater in the Cambridge experiments relative to some of the other facilities.

Thus, it was decided to investigate whether the numerically predicted soil response was sensitive to the influence of higher frequency components in the acceleration trace. In order to do this, an additional FE analysis was run for the case of Motion \#2 but with the input acceleration being generated artificially to meet the specifications exactly. Figure 15 presents a comparison between the experimentally measured acceleration input and the specified ideal motion in both the time and frequency domains. It is confirmed that the measured input motion features additional high frequency components at approximately the third harmonic.

Figure 16 shows the generation of excess pore pressures predicted by the original analyses and with the new idealised input motion. Overall, there is only a slight difference between the dynamic excess pore pressures predicted given the two different input motions, and no discernible difference in the final excess pore pressures post shaking. The slight difference is most noticeable at P1 and the discrepancy reduces with height. Physically, one could expect the soil to effectively filter the high frequencies as a liquefied soil would not respond significantly to the higher frequencies. Thus the majority of displacement, and thus volumetric straining and 
excess pore pressure generation is dominated by the lower frequency components. The possible filtering by the soil of the higher frequencies as the stress waves propagate through the soil could explain the observed reduction in the discrepancy of predicted excess pore pressure generation with height.

Based on the numerical analysis with this constitutive model and the associated numerical damping, it does not appear that the liquefaction behaviour is significantly affected by the $3 \mathrm{~Hz}$ component of the base acceleration apparent in the centrifuge test. Overall, the greater extent of liquefaction observed in the Cambridge experiments relative to other centres is unlikely due to differences in the input acceleration, and may be ascribed to differences in the achieved relative density or degree of saturation of the model.

\section{Conclusions}

In this paper the LEAP-GWU-2015 centrifuge test on a $5^{\circ}$ sloping ground conducted at University of Cambridge is presented. The specific details that were employed during construction and saturation of the centrifuge model are elucidated. During the testing process, the servo-hydraulic actuator imparted small accelerations to the model during transit and it was shown that these accelerations are relatively small and did not result in any significant excess pore pressure generation. The results from the centrifuge test during Motions \#1 \& \#2 are presented along with the simulations from finite element analyses. In the case of Motion \#1 the numerical analysis matched both the acceleration time histories and the lack of excess pore pressure generation seen in the centrifuge test. In the case of Motion \#2, the finite element analysis was able to capture the general increases of excess pore pressure and associated attenuation of the accelerations. However, the de-liquefaction shocks were not replicated by the predicted accelerations. Combined with the prediction of the dynamic excess pore pressure variation, it was concluded the dilatant behaviour of the loose sand at low confining pressures was under predicted for the constitutive parameters chosen. Nevertheless, the numerical analyses replicated many of the salient features of the experimental results and were found to offer additional insight into the boundary effects and deformation mechanism of the slope. The reduced sensitivity of the liquefied soil to the high frequency components in the accelerations, particularly at shallow depths, was also illustrated using the numerical model. Overall, the combination of techniques of centrifuge testing with numerical analyses was found to complement each other well.

\section{References:}

Arulanandan, K., and Scott, R. F. (1993). Verification of Numerical Procedures for the Analysis of Soil Liquefaction Problems, Proc. of VELACS Conference, Davis, California: Balkema, pp. 1-15.

Chan A. H. C. (1988). A generalised fully coupled effective stress based computer procedure for problems in Geomechanics. SWANDYNE User Manual, Swansea

Clough, R.W. and Penzien, J. (1993). Dynamics of Structures, McGraw-Hill Inc., New York

Coelho, P. A. L. F. (2007). In Situ Densification as a Liquefaction Resistance Measure for Bridge Foundations. PhD thesis, University of Cambridge, Cambridge, UK 
Dewoolkar, M. M., Ko, H-Y., and Pak, R. Y. S. (1999). Centrifuge modeling of models of seismic effects on saturated earth structures, Geotechnique, 49(2), pp. 247-266

Haigh, S.K., Ghosh, B. \& Madabhushi, S.P.G. (2005) The effect of time step discretisation on dynamic finite element analysis. Canadian Geotechnical Journal, 42(3) 957-963

Haskell, J. J. M., Madabhushi, S. P. G., Cubrinovski, M. and Winkley, A. (2013). Lateral spreading induced abutment rotation in the 2011 Christchurch Earthquake: observations and analysis, Geotechnique, Vol. 63(15), pp. 1310-1327

Kutter, B. L. (1982). Centrifugal Modelling of the Response of Clay Embankments to Earthquakes. PhD thesis, University of Cambridge, United Kingdom

Kutter, B. L. and Wilson, D. W. (1999). De-liquefaction shock waves, Proceedings 7th US Japan Workshop on Earthquake resistant design of lifeline facilities and countermeasures against liquefaction, Technical report MCEER-99-0019, O'Rourke, T. D., Bardet, J. P., and Hamada, M. (eds), pp. 295-310

Kutter, B. L., Carey, T. J., Hashimoto, T., Zeghal, M., Abdoun, T., Kokkali, P., Madabhushi, S. P. G., Haigh, S. K., Burali d'Arezzo, F., Madabhushi, S. S. C., Hung, W-Y., Lee, C-J., Cheng, H-C., Iai, S., Tobita, T., Ashino, T., Ren, J., Zhou, Y-G., Chen, Y., Sun, Z-B and Manzari, M. T. (2016). LEAP-GWU-2015 Experiment Specifications, Results, and Comparisons submitted to Journal of Soil Dynamics and Earthquake Engineering, Elsevier

Madabhushi S. P. G, Zeng X. (1998). Seismic response of gravity quay walls. II: numerical modelling. Journal of Geotechnical and Geoenvironmental Engineering, ASCE Vol, 124(5), pp. 418-427

Madabhushi, S. P. G., Haigh, S. K., Houghton, N. E. and Gould, E. (2012). Development of a ServoHydraulic Earthquake Actuator for the Cambridge Turner Beam Centrifuge, International Journal of Physical Modelling in Geotechnics, Vol. 12(2), pp. 77-88

Madabhushi, S. P. G., Schofield, A. N. and Lesley, S. (1998). A New Stored Angular Moment (SAM) Based Earthquake Actuator, Proceedings of the International Conference on Geotechnical Centrifuge Modelling 1998, pp. 111-116

Madabhushi, S. S. C. and Madabhushi, S. P. G. (2014). Finite Element Analysis of Floatation of Rectangular Tunnels Following Earthquake Induced Liquefaction, Indian Geotechnical Journal, Vol. 45(3), pp. 233-242

Madabhushi, S.P.G., Houghton, N.E and Haigh, S. K. (2006). A New CNC Sand Pourer for Model Preparation at the University of Cambridge, Proc. of 6th Int. Conf. on Physical Modelling in Geotechnics, ICPMG, Hong Kong

Morris, D.V. (1979). Centrifuge Modelling of Dynamic Behaviour. PhD thesis, University of Cambridge, United Kingdom.

Pastor, M., Zienkiewicz, O. C, Leung K. H. (1985) Simple model for transient soil loading in earthquake analysis. II: non-associative models for sands. International Journal for Numerical and Analytical Methods in Geomechanics, Vol. (9) pp. 477-498 
Schofield, A.N. (1981). Dynamic and earthquake geotechnical centrifuge modelling, Proceedings of the International Conference on Recent Advances in Geotechnical Earthquake Engineering and Soil Dynamics, St Louis, Vol. 3, pp. 1081-1100

Semblat, J.F., Brioist, J.J., and Luong, M.P. (1999). Mean stress dependent damping of seismic waves in sand, Proc. Earthquake geotechnical engineering, Edited by P. Sêco e Pinto. A.A. Balkema, Rotterdam. pp. 95-100

Stringer, M. E. and Madabhushi, S. P. G. (2009). Novel Computer-Controlled Saturation of Dynamic Centrifuge Models using High Viscosity Fluids, Geotechnical Testing Journal, Vol. 32(6), pp. 53-59

Zienkiewicz, O. C., Chan, A. H. C., Pastor, M., Schrefler, B. A., Shiomi, T. (1999). Computational Geomechanics with Special Reference to Earthquake Engineering, John Wiley, New York, 1999

\section{Figures}

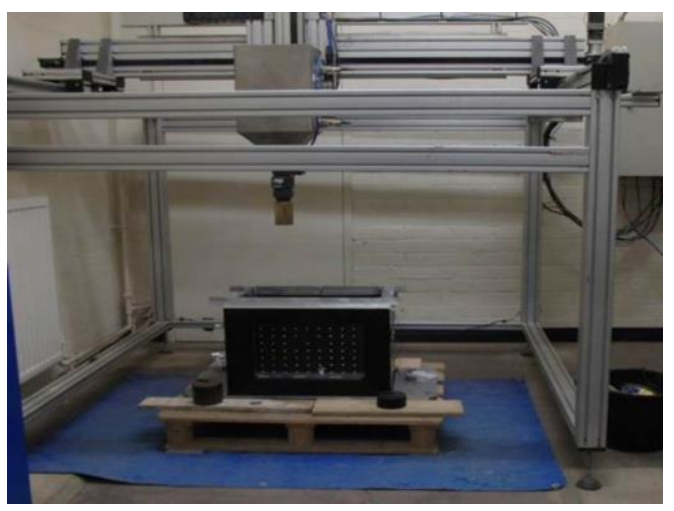

Figure 1: Automatic sand pouring system

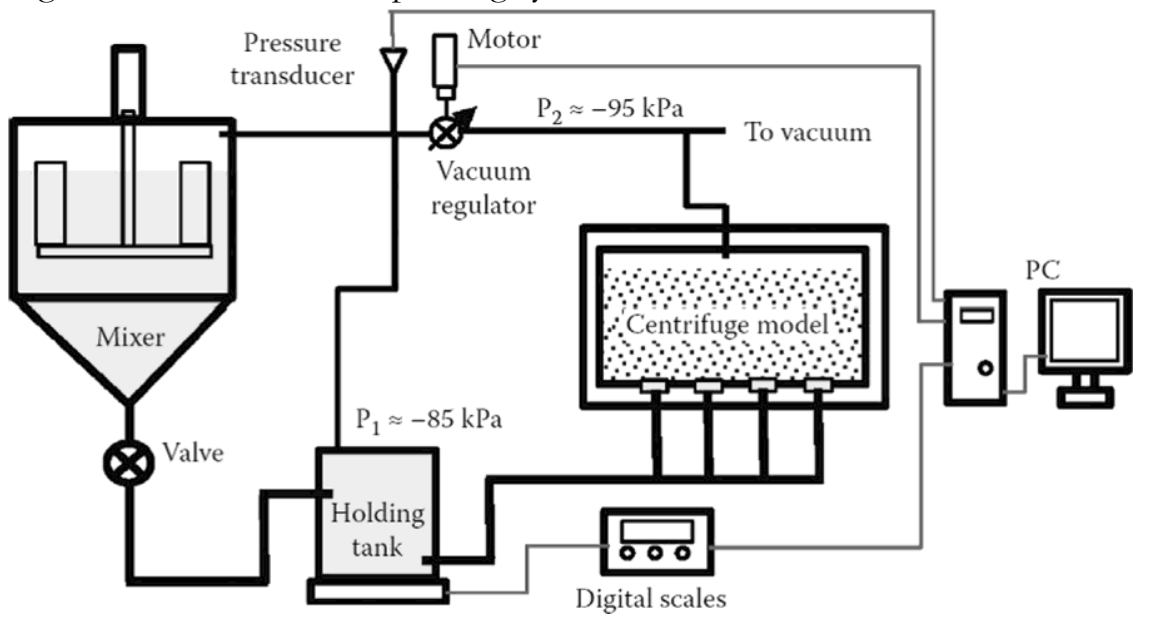

Figure 2: Schematic layout of the CAM-SAT system 


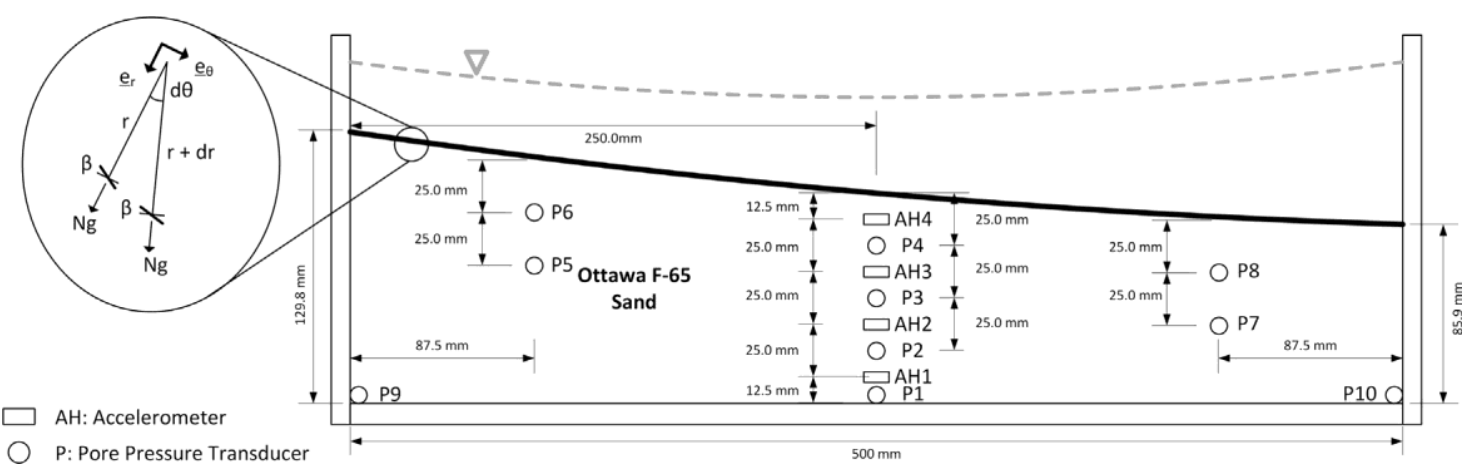

Figure 3: Schematic cross-section of the Cambridge model slope

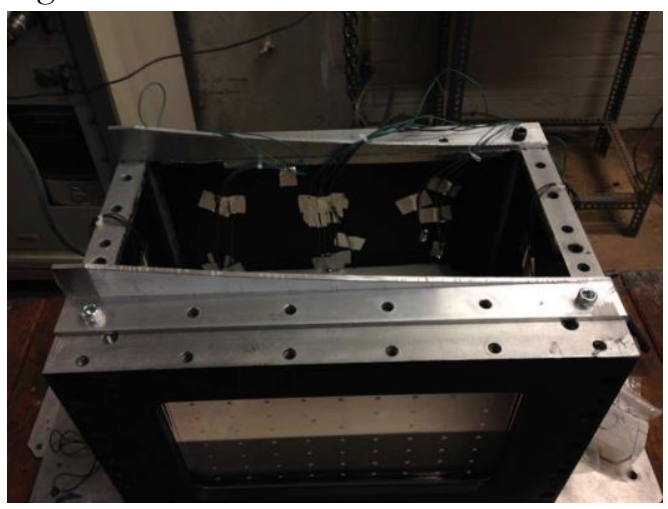

Figure 4: Logarithmic guide plates on model container
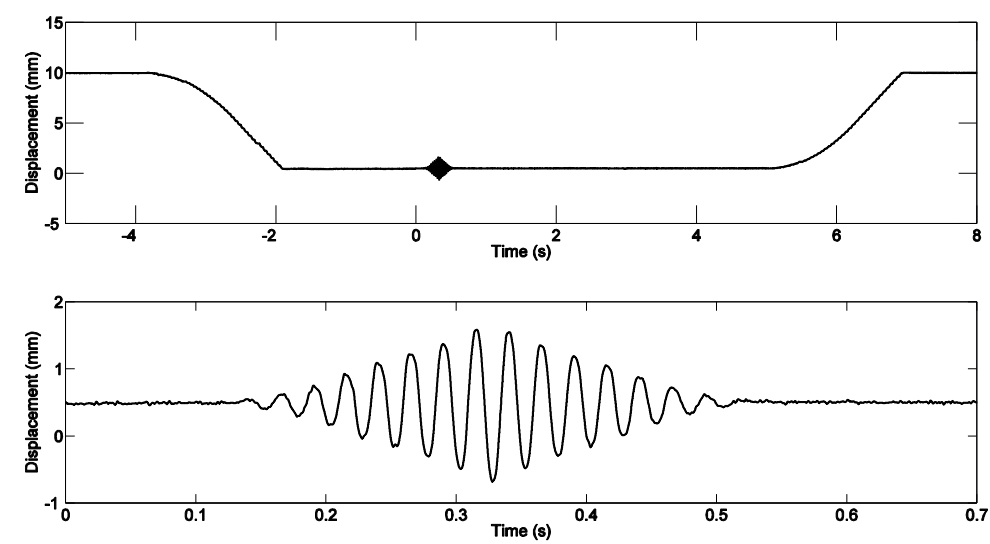

Figure 5: Transit of the servo-hydraulic actuator before and after the earthquake event
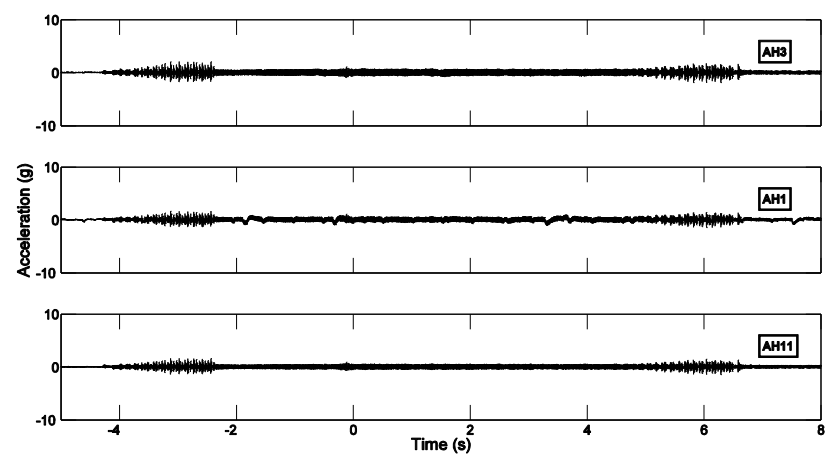

a) Motion 1 

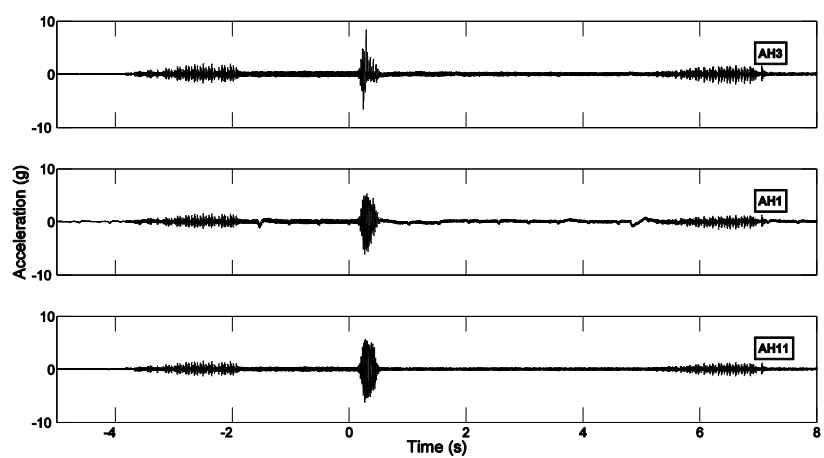

b) Motion 2

Figure 6: Accelerations during the transit phase
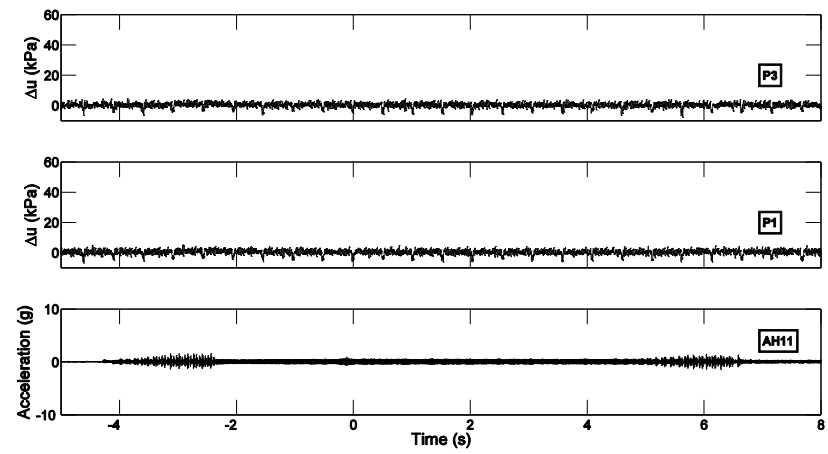

a) Motion 1
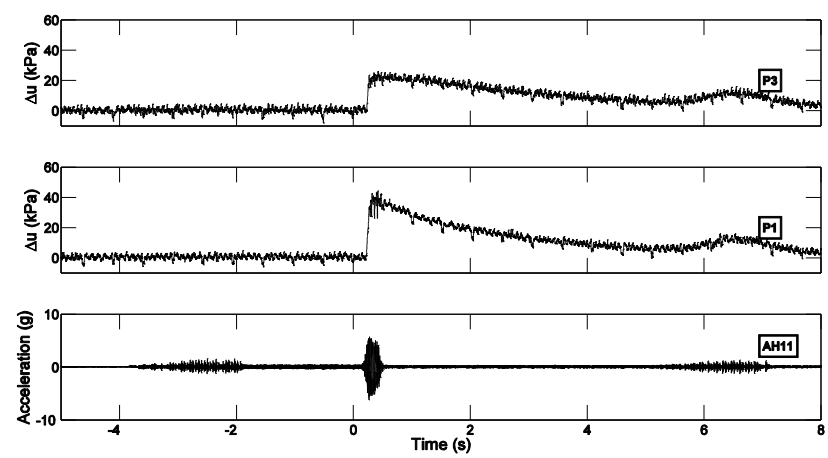

b) Motion 2

Figure 7: Excess pore pressures generated during transit phases

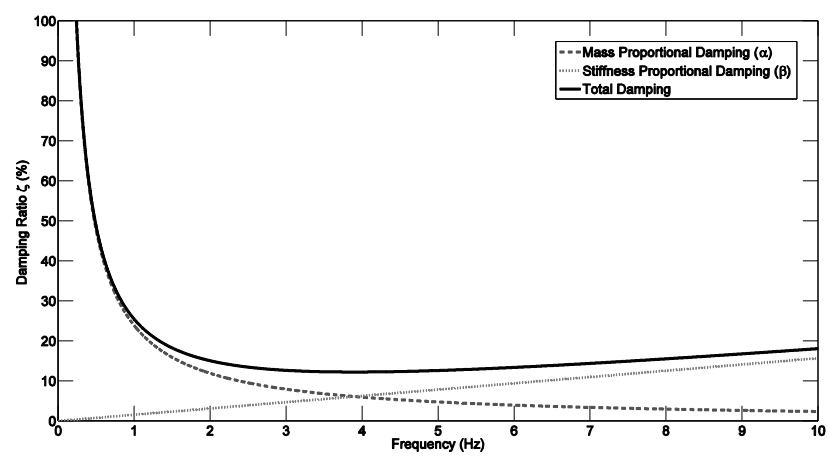


Figure 8: Selection of damping parameters

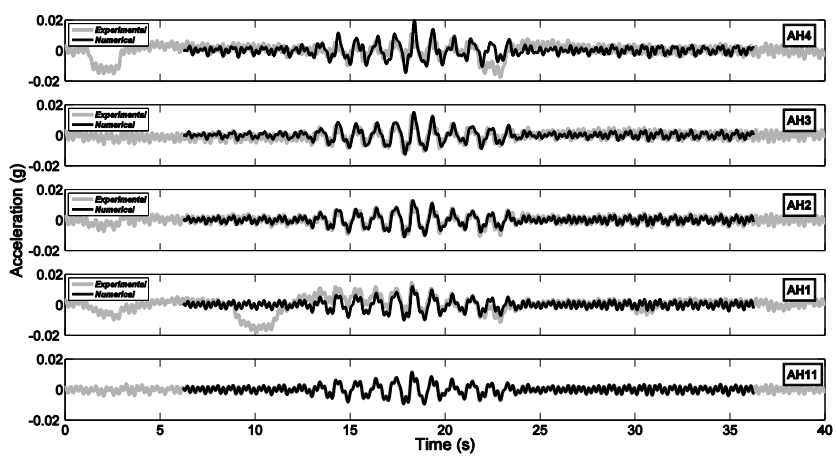

Figure 9: Accelerations during earthquake motion 1

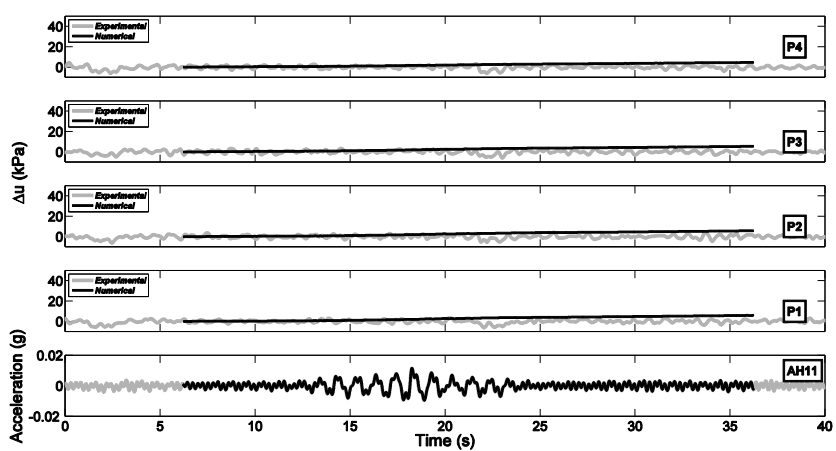

Figure 10: Excess pore pressures during earthquake motion 1

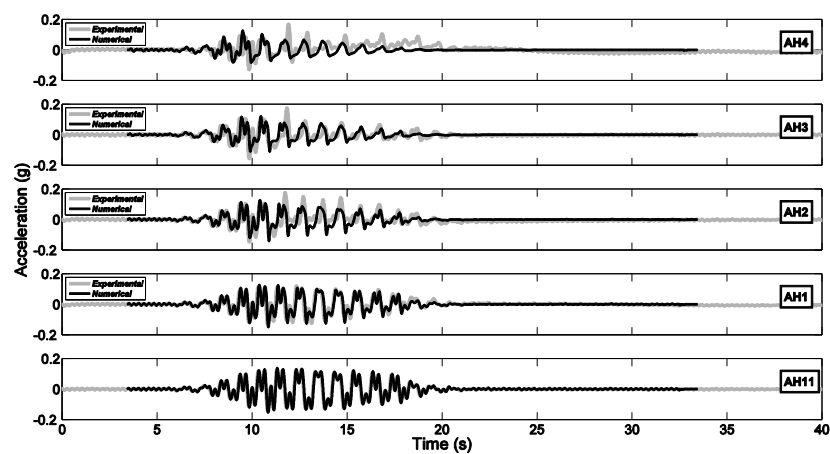

Figure 11: Accelerations during earthquake motion 2

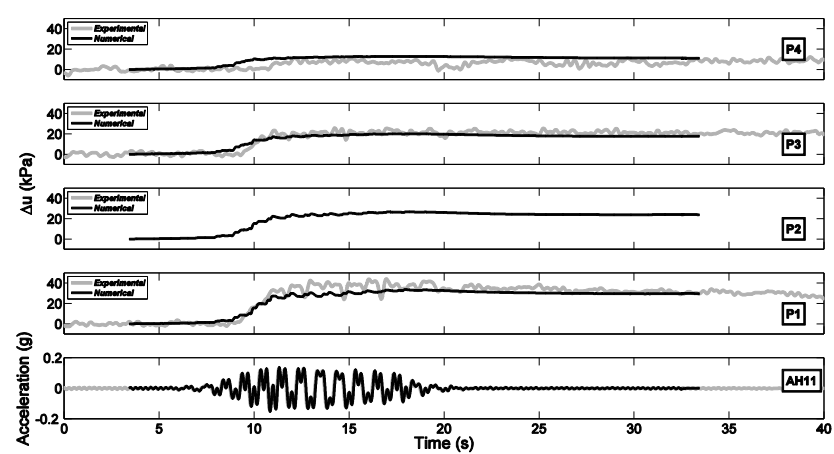

Figure 12: Excess pore pressures during earthquake motion 2 


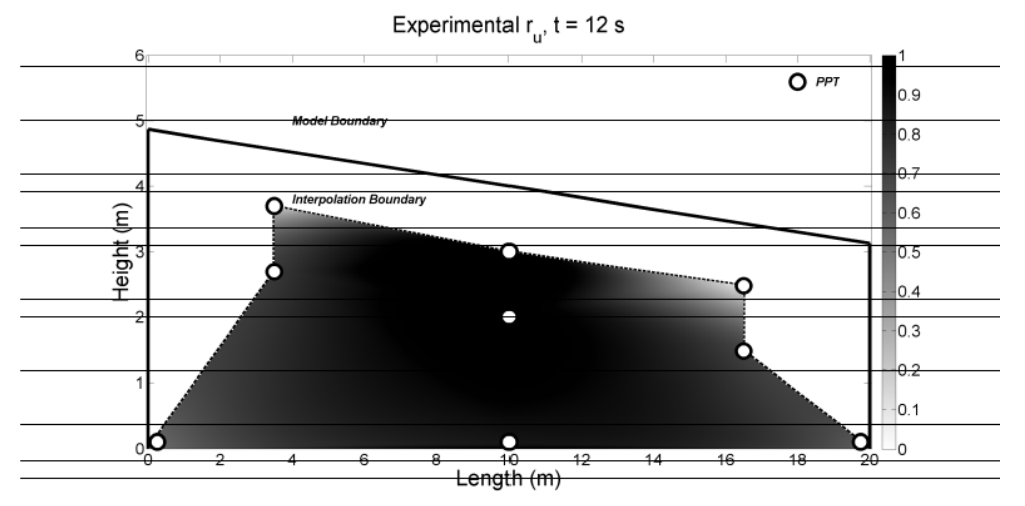

i. Experimental Contours

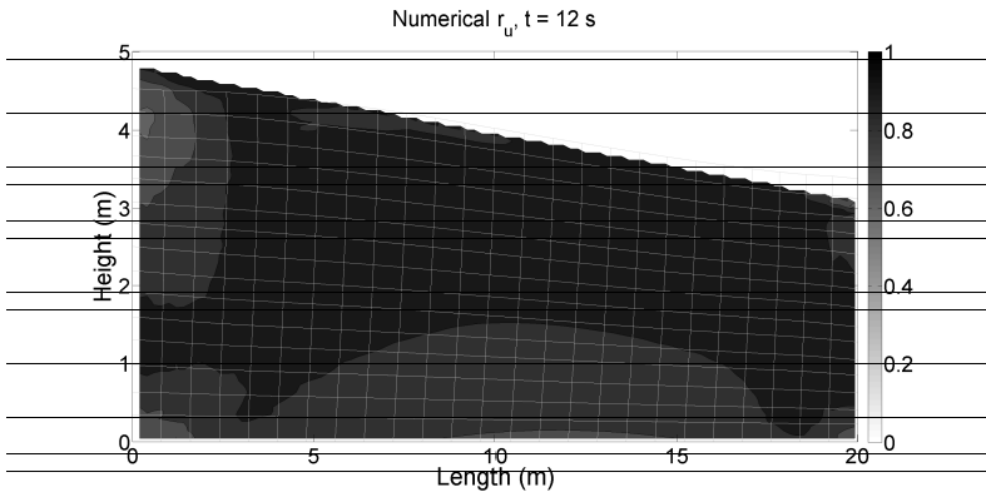

ii. Numerical Contours

Figure 13a: Contours of excess pore pressure ratio at $t=12 \mathrm{~s}$

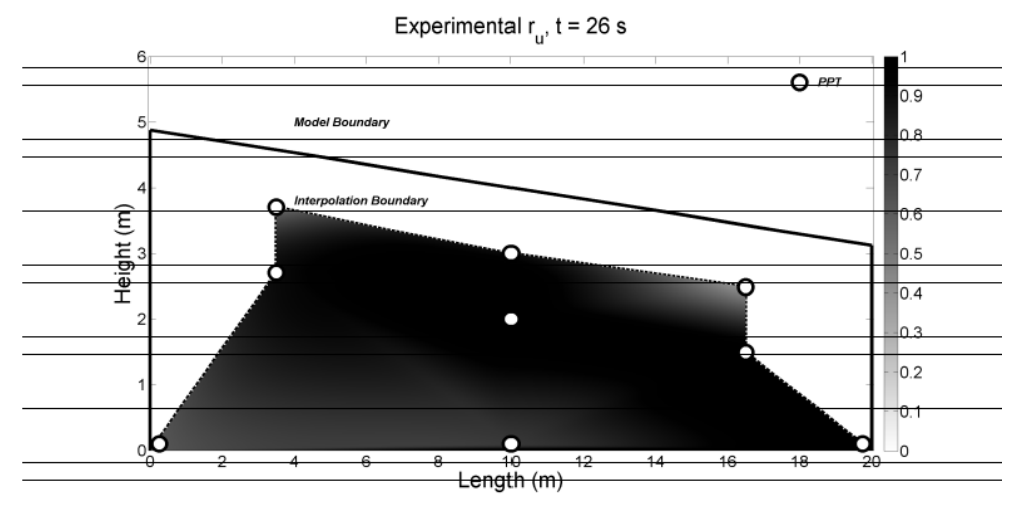

i. Experimental Contours

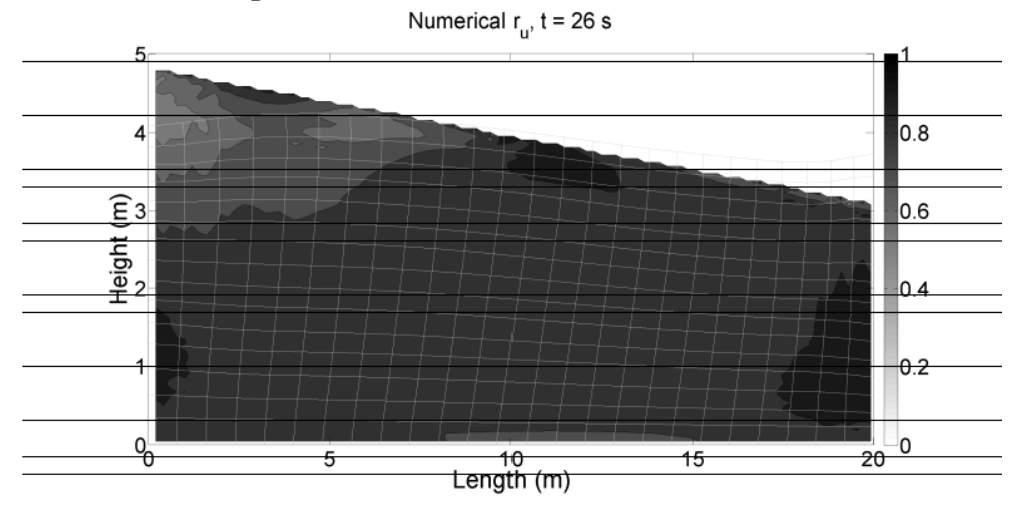

ii. Numerical Contours

Figure 13b: Contours of excess pore pressure ratio at $t=26 \mathrm{~s}$ 


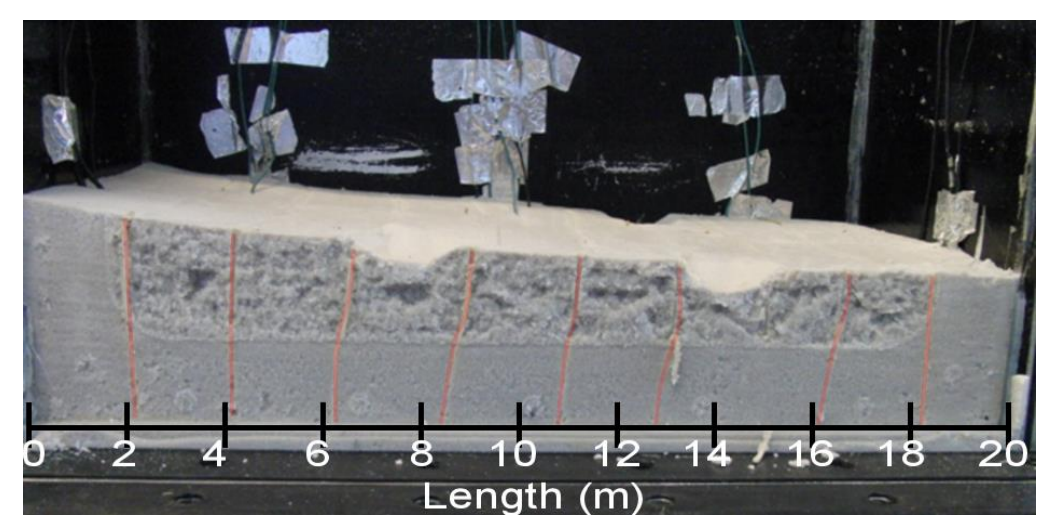

Figure 14a: Experimentally observed lateral deformations in the slope

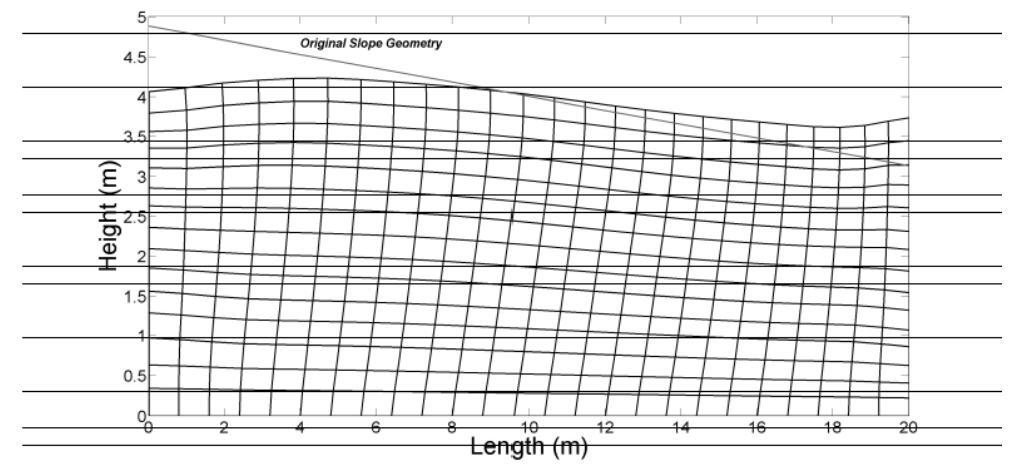

Figure 14b: Numerically predicted lateral deformations in the slope
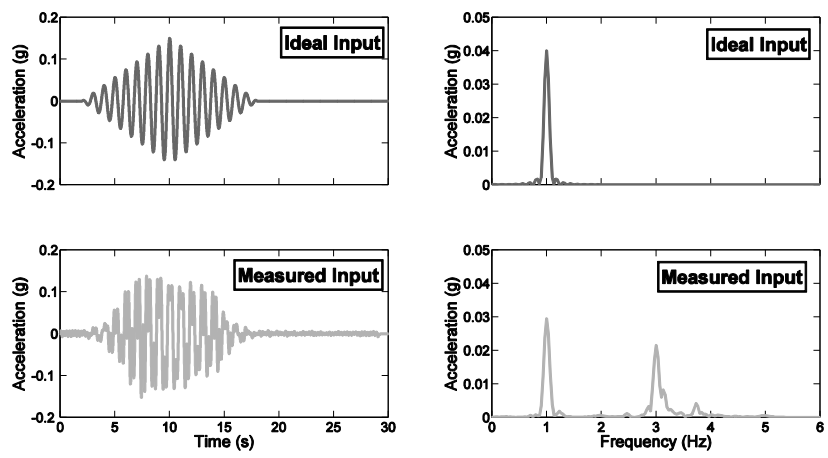

Figure 15: Comparison between the ideal and measured input motion

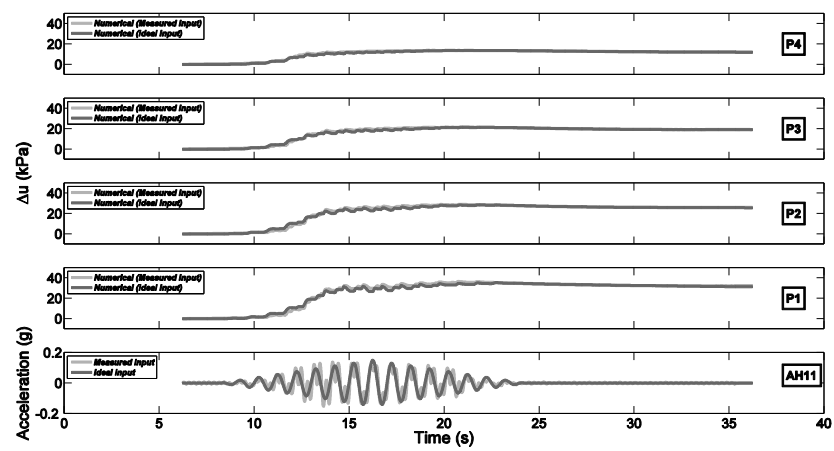

Figure 16: Comparisons of predictions for excess pore pressures using ideal and measured input motions

\begin{tabular}{|l|l|l|}
\hline Description of the parameter & Symbol & Value \\
\hline Slope of critical state line for the yield surface & $\mathrm{M}_{\mathrm{f}}$ & 1.164 \\
\hline Slope of the critical state line for plastic & $\mathrm{M}_{\mathrm{g}}$ & 0.97 \\
\hline
\end{tabular}




\begin{tabular}{|l|l|l|}
\hline potential & & \\
\hline $\begin{array}{l}\text { Parameter to determine dilatancy } \\
\text { using stress ratio for loading vector }\end{array}$ & $\alpha_{\mathrm{f}}$ & 0.45 \\
\hline $\begin{array}{l}\text { Parameter to determine the dilatancy using } \\
\text { stress ratio for plastic strain vector }\end{array}$ & $\alpha_{\mathrm{g}}$ & 0.45 \\
\hline Initial Bulk Modulus (MPa) & $\mathrm{K}$ & 19 \\
\hline Shear Modulus (MPa) & $\mathrm{G}$ & 3.5 \\
\hline Plastic modulus on loading (Pa) & $\mathrm{H}_{\text {oloading }}$ & 200 \\
\hline Plastic modulus on unloading (MPa) & $\mathrm{H}_{\text {ounloading }}$ & 400 \\
\hline Reference value of effective stress (kPa) & $\mathrm{p}^{\prime}$ & 25 \\
\hline Powers on Stiffness Variation & $\mathrm{p}_{\mathrm{f}, \mathrm{p}_{\mathrm{g}}}$ & 0.5 \\
\hline Shear hardening parameter 1 & $\beta_{\mathrm{o}}$ & 4.2 \\
\hline Shear hardening parameter 2 & $\beta_{1}$ & 0.2 \\
\hline $\begin{array}{l}\text { Parameter for plastic deformation } \\
\text { during unloading }\end{array}$ & $\gamma_{\mathrm{Hu}}$ & 2 \\
\hline $\begin{array}{l}\text { Parameter for plastic deformation } \\
\text { during reloading }\end{array}$ & $\gamma_{\mathrm{DM}}$ & 0 \\
\hline
\end{tabular}

Table 1 Constitutive Parameters for PZ Mark III model 